\title{
Compressive and Shear Analysis of Rubber Block Under Large Strain
}

\author{
${ }^{1}$ K. Sridharan and ${ }^{2}$ R. Sivaramakrishnan \\ ${ }^{1}$ Department of Rubber and Plastics Technology, \\ ${ }^{2}$ Department of Production Technology, \\ Anna University MIT Campus, Chennai-600 044, India
}

Received 2013-01-11, Revised 2013-06-13; Accepted 2013-07-02

\begin{abstract}
The Elastomeric materials have found use in a wide range of applications, including hoses, tires, gaskets, seals, vibration isolators, bearings and dock fenders. The analysis of rubber blocks for its compression and shear behavior has been carried out using the imaging techniques. The dynamic stressing and its associated change in shape of the rubber blocks during large compression are very limited as their measurements were difficult. A newly developed Machine Vision based image processing test has been effectively used to study the deformation characteristics of the rubber blocks under large strains. An extended analysis on the rubber blocks has been carried out to understand the compression and deformation behavior in static and dynamic condition and the nonlinear behavior were also characterized. The rubber blocks of distinguished geometries have shown diverse change in shape and nonlinear deformation behavior under compression/shear loading.
\end{abstract}

Keywords: Rubber Blocks, Nonlinear, Static, Dynamic

\section{INTRODUCTION}

Friction force between the rubber and a surface has two contributions commonly described as the adhesion and hysteretic components. Several theories have been developed to describe surface roughness and the relation to the real contact area. The study of compressive behavior of rubber blocks and its shape change has its significance in many engineering applications. Many papers have been reported on the compression of rubber blocks between rigid plates and the linear elastic strains have been validated by the researchers. Here, an attempt has been made to understand the large compressive strain phenomenon along with linear elastic strain. When the block has been compressed they exhibit a nonlinear stress distribution and dramatically different behaviors predicted by earlier investigator (Gent, 1994). A number of works had been carried by Gent (1994) for the development of nonlinear elastic solution for the deforming rubber block to evaluate the non linear response.
Rubber does not obey the classical law of friction that states that the ratio between frictional force and normal load is a constant known as coefficient of friction $(\mu)$. For rubber, $\mu$ decreases rapidly as the velocity increases. The friction coefficient of rubber is also affected by the existence of lubricants or water and thus the effect of pressure distribution and frictional force is yet to be clarified. The mechanism of optimized rubber block contacted on a dry surface was different from the wet surface and its friction increased in dry surface and decreased in wet surface (Nakajima and Takahashi, 2002).

When the natural rubber cylindrical blocks had been compressed the contact area increased substantially and a nonlinear distribution was observed at all strain levels (Patenaude et al., 2005). The traction forces generated by a rolling pneumatic tyre are influenced by a number of factors out of which tyre construction, tread pattern, tread material are in the interest of tyre designer (Veirh, 1992). The contact time of a rubber tread block for a Corresponding Author: K. Sridharan, Department of Rubber and Plastics Technology, Anna University MIT Campus, Chennai-600 044, India 
vehicle velocity of $100 \mathrm{~km} \mathrm{~h}^{-1}$ takes about $5 \mathrm{~ms}$, during this contact time the tread block passes a sticking and a sliding phase and finally snaps out (Moldenhauer and Kroger, 2010).

In the complete absence of slippage, stress and strain are not uniform throughout the test piece and barreling takes place on compression. In real cases during the dynamic condition the increase in temperature makes rubber more elastic which results in stronger stress concentration and causing cracks at the contact tips at faster rate. The contact between tyre and road has been observed at a very high magnification and the real area of contact monotonically decreased (Persson, 2009). The tyre tread blocks are stable enough to provide its own performance towards mileage, traction, low noise and heat built up properties. The contact pressure is not uniform in its functioning and it had been reported that the friction force is maximized when the pressure distribution is uniform from the experiments conducted on the optimized rubber blocks to increase the frictional force by uniform contact pressure distribution The tread blocks are subjected to both normal as well as lateral forces which make them to suffer under uneven pressure distribution.

\subsection{Review on Friction and Pressure Distribution}

Compression set measurements have been used traditionally as an indication of sealing performance because they are relatively simple tests to perform, to know what correlation exists between compression set and compression stress relaxation. Measurement of set under compression provides a practical evaluation of the creep or the stress relaxation of rubber and very useful for the purpose where a high degree of precision is not required. Several theories have been developed to describe surface roughness and the relation to the real contact area. It is a known factor that friction is due to energy dissipated when rubber is compressed and released by asperities. There are four different sources of non-linearity in solid mechanics which includes geometrical, material, force boundary conditions and displacement boundary conditions. In the tyre tread block contact phenomenon, the above stated nonlinearity is highly concerned as they are not constant and its variation is highly dependent on pavement. One basic part of the tyre is the tread pattern which is in direct contact with the road surface and thus it is responsible for the friction level of the whole tyre.
The tread pattern is a source of anisotropy in tyre tread layer. Tread blocks may be compressed and bent so much that the vertical load is transmitted just by frontal edges of the tread pattern blocks. Lateral displacements of the surface of tread layer become substantially greater than those in radial directions due to considerable bending and stretching of tread blocks. When the tyres are in static, the distribution of the normal load $F$ is symmetric with respect to the center of the contact patch. However, when the tyres are rotating, the normal load distribution is non-symmetric. The study of tread block behavior under the static and dynamic condition would be effective and more useful in the pattern design and analysis.

It is one of the major objectives of the research to understand the compression behavior of the rubber blocks and its effect on shape factor under variable loading condition in the process of designing a rubber component. The general behavior of a tyre tread block has been taken into consideration for this study and analysis of the same has been carried out. Tread blocks are compressed and bent so much that the vertical load is transmitted just by frontal edges of the tread pattern blocks. Lateral displacements of the surface of tread layer become substantially greater than those in radial directions due to considerable bending and stretching of tread blocks. The present work focuses on the modeling of the tread block with a different geometry and to analyze its compression and deformation characteristics using the developed image tool.

The static loading of the cylindrical shaped rubber block has been studied in past and its response have been reported in papers. Attempts have been made to study the dynamic response of the cylindrical and other geometrical shaped rubber block under large compressive/shear strains. Experiments have been conducted on the rubber tread block with same contact area and different geometrical shapes i.e., same volumetric properties and mass properties but with different shapes. The friction coefficient was taken to be constant throughout the analysis.

\section{MATERIALS AND METHODS}

The working setup is made rigid for better stability of the system. A camera stand of adjustable type for holding the camera is used and the camera can be adjusted to move up and down in order to capture the image. An Acrylic board is placed over the stand which acts as a fixed platen and the blocks are compressed by the piston attached in the double acting cylinder. 


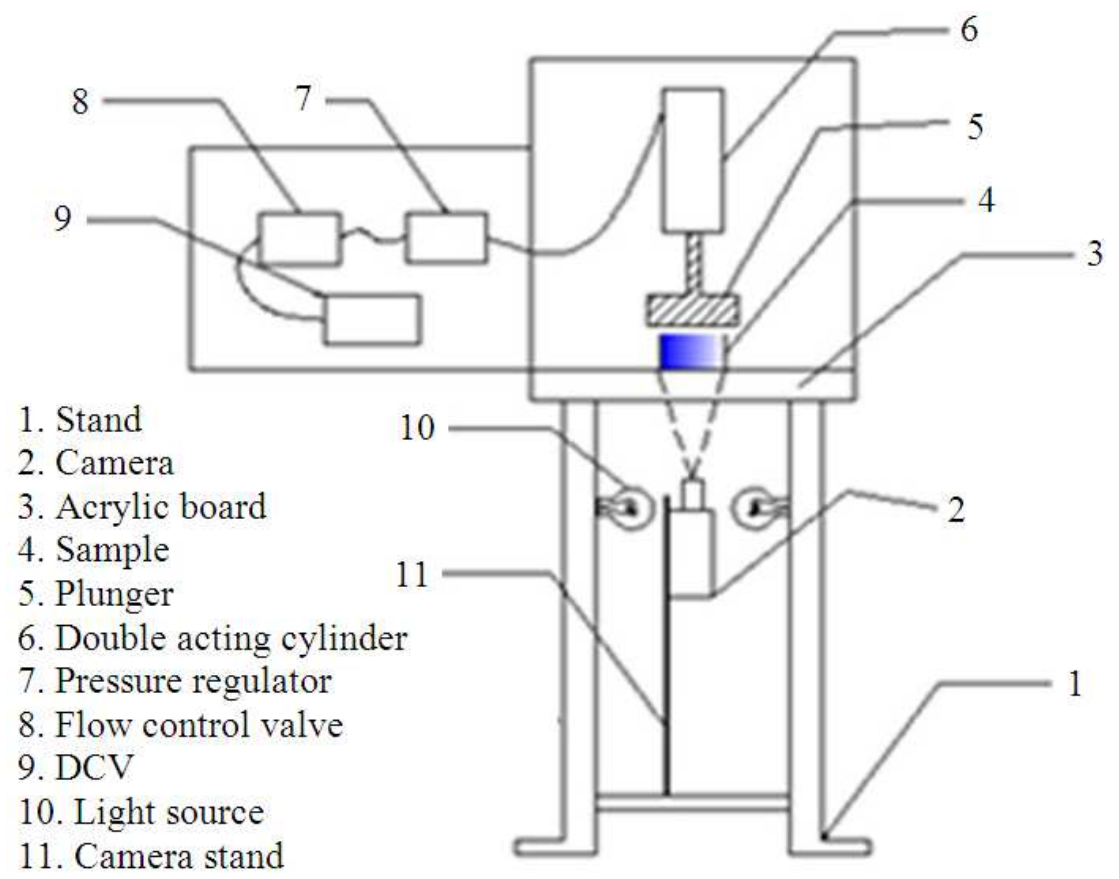

Fig. 1. Schematic of experimental setup
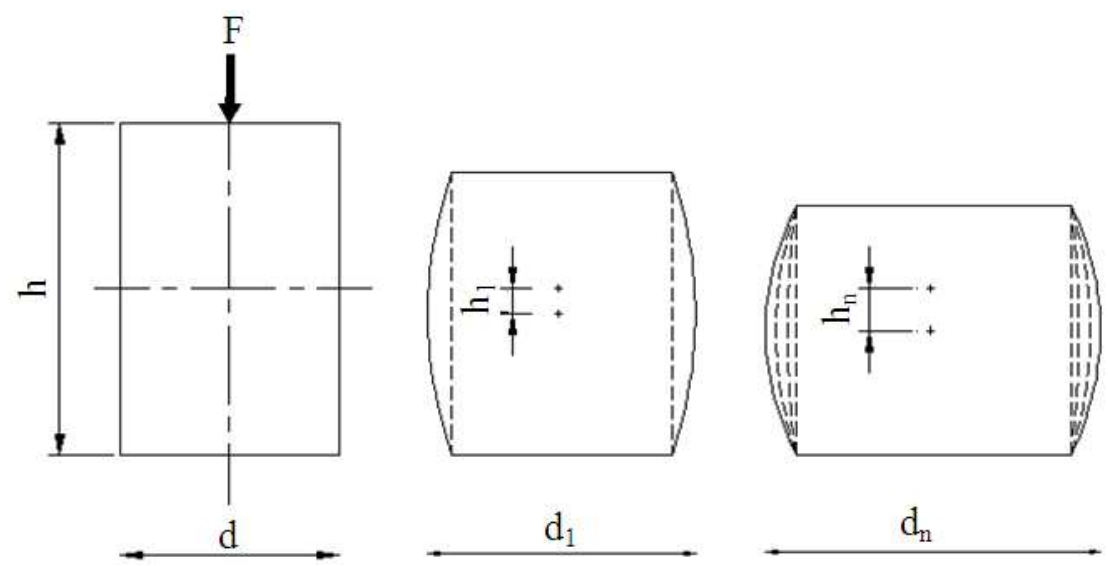

Fig. 2. Deformation pattern of cylindrical rubber block under normal compressive force

A pneumatic control setup with FRL unit and pressure gauge are mounted in series for its effective functioning. The piston compresses the rubber block under variable pressure load and the stiffness were evaluated. A Samsung SDC 310Analog camera is fixed in the camera stand and is connected with a computer in order to view and capture the image. Effective lights sources have been provided for better illumination. The Fig. 1 given below shows a complete experimental set up used in this investigation.

\subsection{Experimental Investigation}

The basic idea to conduct the research is to develop a machine vision tool to study the compression and deformation behavior of rubber blocks. The rubber blocks of different geometrical shapes which are similar to the tread blocks of the tyres were analyzed in static and dynamic condition. The deformation behavior of the block has been studied and will be useful to optimize the tyre design. The deformation behavior of a regular 
cylindrical, square and other geometrical block is studied under different compression loads. The cylindrical rubber samples of diameter $16 \mathrm{~mm}$ and of height $10 \mathrm{~mm}$ (aspect ratio $\mathrm{a} / \mathrm{h}=0.8$ ) were used in this study. The pressure range between 1 bar to 5 bar was applied over the cylindrical rubber block to compress it and resulting compressive/shear response was recorded. The Fig. 2 clearly illustrates the increase in compression pressure has its definite effect over the change of shape in the rubber tread block. Analysis of the rubber blocks proves that for the same contact area and volume, a distinguished performance under the dynamic compression test carried out in the laboratory. Similar test was also extended to the rubber block of other geometrical shapes.
The compression studies on the rubber block have been carried out experimentally and its characteristics had been studied. It had been inferred from the past researches carried out on the rubber blocks that the geometric shape has its influence over the friction and wear on the contact patch (Gabel and Moldenhauer, 2008). The compression and recovery of the block under the dynamic condition includes a fatigue in the blocks. It has been found in the experimental work the circular and square shaped blocks uniformly bulges outward for the applied normal force and the shape change also progressively increases on increasing the magnitude of the force as shown in the Fig. 2. In the case of the other geometrical shaped rubber blocks i.e., the convex and concave edged blocks the bulging arise in a distinguished way and further studies are carried out.

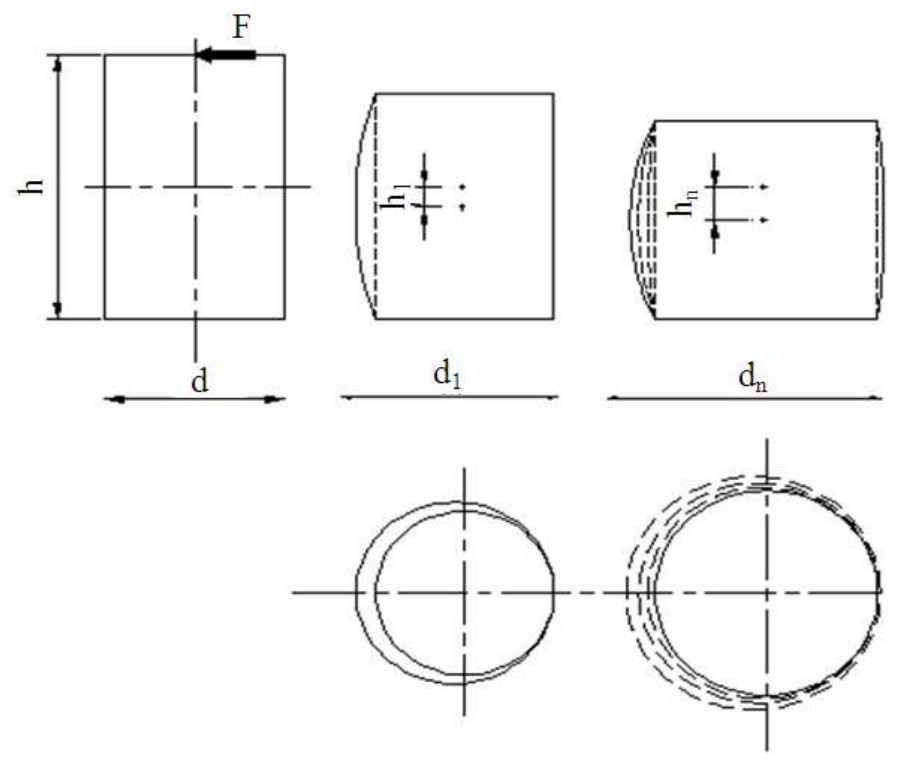

Fig. 3. Deformation pattern of cylindrical rubber block under shear force

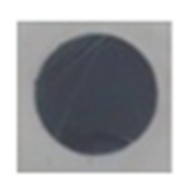

$\mathrm{P}=0 \mathrm{bar}$

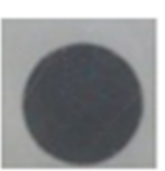

$\mathrm{P}=0$ bar

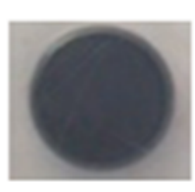

$\mathrm{P}=1$ bar

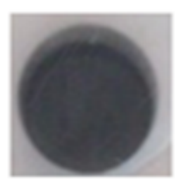

$\mathrm{P}=1 \mathrm{bar}$

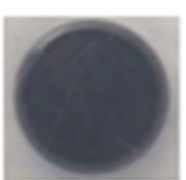

$\mathrm{P}=2$ bar

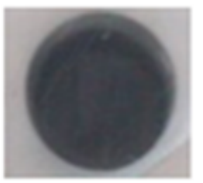

$\mathrm{P}=2$ bar

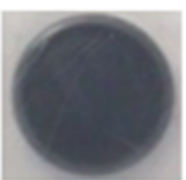

$\mathrm{P}=3$ bar

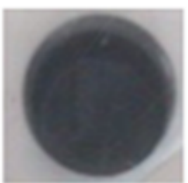

$\mathrm{P}=3$ bar
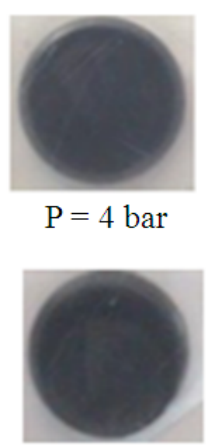

$\mathrm{P}=4$ bar

Fig. 4. Un-deformed and deformed images of cylindrical rubber block for applied compressive/Shear force at various strain levels 
On the compression of cylindrical rubber block it is observed that the reduction in height $\mathrm{h}$ of the block is progressively decreased by increasing the normal load $\mathrm{F}$ which is proportional to the increase in diameter. The barreling effect of the rubber block on compression was proportional to the normal force and the barrel diameter increased significantly for the applied compressive load. The studies have been extended for the shear force by changing the setup and the force has been applied to the piston by tilting it to the required angle in the setup. It has been observed that the shear behavior shows a distinct variation from the compression behavior for the same magnitude of applied force. The friction behavior of the rubber tends the block to shear out from inward to outward direction of the applied force creating an unsymmetrical barreling effect. It could have been inferred from the Fig. 3 shown below that the compression and shear behavior shows a distinct variation in shape change of a regular axi-symmetric cylindrical block. In case of the shear load, the expansion of the block began to open widely towards the outer edge including more distortion in the direction of applied force and the corresponding shape change has been shown in the Fig. 3. The investigations have been proven that the compressive/shear viscoelastic response of the rubber materials shows a distinct nonlinear deformation in application. One could have been easily understand the linear elastic properties and its behavior on the viscoelastic material under static conditions but, the dynamic response have not been clarified which includes both the compressive and shear action. As the tread block suffers by both compression/shear forces in on-road conditions, their study should be approached by considering both the forces. The cylindrical rubber block shows comparatively a large deformation and increase in area for the same magnitude of shear forces than the compressive force.

In many Engineering applications such bridge bearings, tyre tread blocks, dampers, mountings. both component of compressive/shear force has its own effects. Thus the study of shape change to the applied load would enhance the knowledge of designer to optimize the necessary function of the rubber components. In complete absence of slippage, stress and strain are not uniform throughout the test piece and barreling takes place on compression. The images of the circular shaped rubber blocks under the compressive/shear force are shown in the Fig. 4. The above figure depicts a distinguished variation in the change in bulge area for the same magnitude of applied compressive/shear force and a nonlinear deformation behavior.

\section{RESULTS AND DISCUSSION}

For tires, high friction levels are needed to provide good directional control for vehicles. The dynamic interaction between the tyre and road is very complex to understand. The tread block is the only tyre component that is in direct contact with road surface. Due to the rolling motion of tyres, the contact of individual tread block has its own significance in contact events. Thus by conducting compressive/shear studies on the tread block and understanding its diverse change in shape would be vital for optimized tyre design. The Fig. 5 and 6 depicts the change in central bulge area as the function of contact pressure under the normal compressive/shear load. The deformation behavior was found to be distinguished for the same contact area and same magnitude of applied force. It has also been inferred that the shape change was comparatively large than the normal load and the barreling effect was also not symmetric and thus shear force creates a distinguished behavior in the rubber blocks on its application. It has been observed that for a rubber block under a large shear force showed a barreling on the direction opposite to the applied force. Thus the rolling tyre phenomenon is more complicated to understand this analysis carried out on geometrical shape change of the tread blocks would be more effective in tyre design.

The circular shaped blocks uniformly bulges outward for the applied normal force and the shape change also progressively increased on increasing the magnitude of compressive force. Under the shear force of same magnitude the block shear out from inward to outward direction for the applied force creating an unsymmetrical barreling effect. Thus the rolling motion of the tyre creates a dynamic stress over the tread block and its shape parameter studies would improve the designer for optimizing its performance. Furthermore, this procedure was applied for complicated block shapes and was verified to optimize the tyre design. The image processing experimental setup with all accessories has been presented in the Fig. 7. 
K. Sridharan and R. Sivaramakrishnan / American Journal of Applied Sciences 10 (7): 681-687, 2013

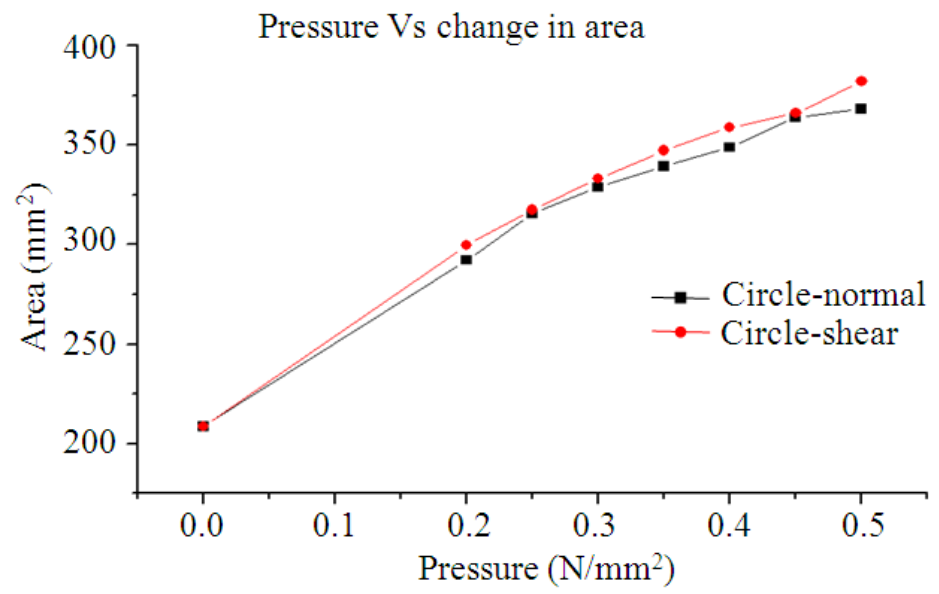

Fig. 5. Change in bulge area as the function of applied pressure load for the circular shaped rubber blocks under compressive/shear forces

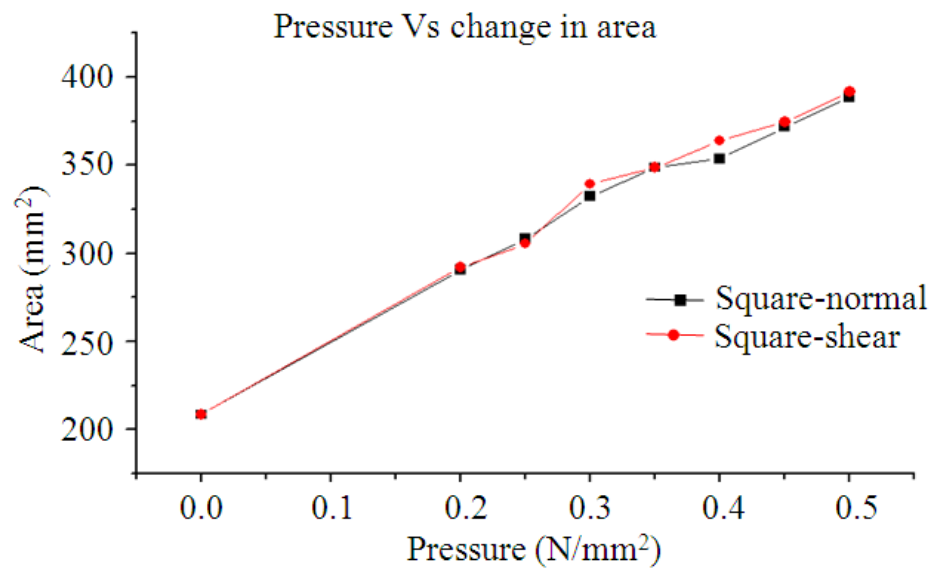

Fig. 6. Change in bulge area as the function of applied pressure load for the Square shaped rubber blocks under compressive/shear forces

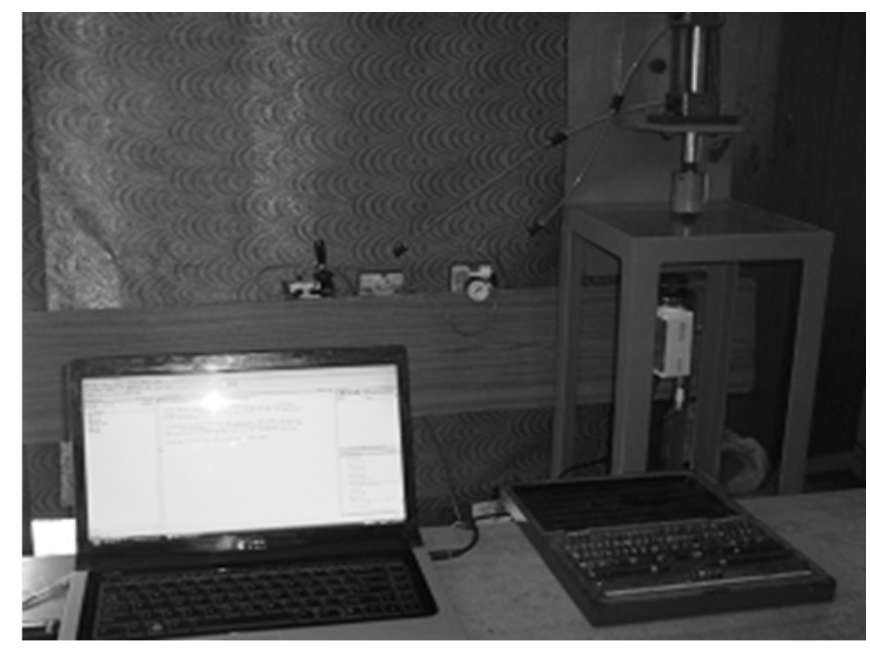

Fig. 7. Experimental setup 


\section{CONCLUSION}

The dynamic interaction between the rubber block and a rigid body is very complex as the rubber behavior is highly non-linear. There is substantial increase in the contact area of the rubber blocks which are distinguished in the compressive and shear loads. The rubber blocks of cylindrical shape shows a symmetric barreling under compression for all loads. When the same approach has been extended to the shear analysis, the barreling effect is high on the direction of the force applied and shown a small barreling on the load free side for a very high shear load. The square block shown almost similar behavior in the compression but it behaved in distinguished way under shear force. The other chosen geometrical rubber blocks behavior will be further well analyzed and reported in the future. It has been inferred that for a same contact area, mass and volumetric properties of the rubber block of different geometrical shapes in compression/shear studies under large compressive strain shows a distinguished behavior.

\section{REFERENCES}

Gent, A.N., 1994. Compression of rubber blocks. Rubber Chem. Technol., 67: 549-558. DOI: 10.5254/1.3538691

Gabel, G. and M.K. Moldenhauer, 2008. Local effects between the tyre and the road. ATZautotechnology, 8: 48-53. DOI: $10.1007 / \mathrm{BF} 03247047$

Moldenhauer, P. and M. Kroger, 2010. Simulation and experimental investigations of the dynamic interaction between tyre tread block and road. Elastomer Friction, 51: 165-200. DOI: 10.1007/978-3-642-10657-6_6

Nakajima, Y. and F. Takahashi, 2002. Increase of frictional force of rubber block by uniform contact pressure distribution and its application to tire. Rubber Chem. Technol., 75: 589-604. DOI: 10.5254/1.3544986

Patenaude, K.J., Z. Tao, J.L. Mead and R.G. Stacer, 2005. Response of elastomeric blocks during large compression strains. Rubber Chem. Technol., 78: 188-198. DOI: $10.5254 / 1.3547877$

Persson, B.N.J., 2009. Theory of powdery rubber wear. J. Phys. Condens Matter, 21: 1-8. DOI: 10.1088/0953-8984/21/48/485001

Veirh, A.G., 1992. A review of important factors affecting treadwear. Rubber Chem. Technol., 65: 601-658. DOI: $10.5254 / 1.3538631$ 